\title{
Maximum Likelihood Estimation, Interpolation and Prediction for Fractional Brownian Motion
}

\author{
Rachid Harba ${ }^{1}$, Hassan Douzi ${ }^{2}$, and Mohamed El Hajji ${ }^{2}$ \\ ${ }^{1}$ Laboratoire PRISME, Polytech'Orléans, Université d'Orléans 45067 Orléans, France \\ Rachid.Harba@univ-orleans. fr \\ ${ }^{2}$ Laboratoire IRF-SIC, Faculté des sciences, Université Ibn Zohr, BP8106 Agadir, Maroc \\ \{douzi_h, hajjimohmed\} @yahoo.fr
}

\begin{abstract}
The maximum likelihood (ML) estimation approach for fractional Brownian motion $(\mathrm{fBm})$ is explored in this communication. First, a ML based estimation of the $\mathrm{H}$ parameter is implemented on the signal itself. This approach on the signal itself can easily be applied on non-uniformly sampled data or directly useful in the case of incomplete data. Secondly, the method is extended to provide a ML prediction and a ML interpolation for $\mathrm{fBm}$ which could be of interest in many domains. Results also help to explain errors in other interpolating methods such as the midpoint displacement algorithm used to synthesize fBm data.
\end{abstract}

\section{Introduction}

Fractional Brownian motion ( $\mathrm{fBm}$ ) of $\mathrm{H}$ parameter in the range $] 0 ; 1$ [ is defined as an extension of Brownian motion [1]. One of the main issues when dealing with such data is to estimate the $\mathrm{H}$ parameter [2-3]. Among the numerous methods to achieve such a goal, the maximum likelihood (ML) approach proposed by Lundahl et al. [4] is often used due to its asymptotical efficiency [5]. It is also efficient in noisy environments [6]. But the ML based estimation of the $\mathrm{H}$ parameter is performed on the $\mathrm{fBm}$ increments which may be a limiting factor in some cases.

Here, we propose an ML estimate of the $\mathrm{H}$ parameter processed on the $\mathrm{fBm}$ itself. This allows direct extension of the method to include cases where there may be irregular sampling or incomplete data. Moreover, an ML based prediction and interpolation technique for a fBm signal easily result.

This communication is organized as follow. In the next section, fBm is defined and its main properties are derived. Then, the ML based estimation of the $\mathrm{H}$ parameter is achieved and is tested on exact fBm data. Finally, ML interpolation and prediction are presented and a real data example illustrates the methods.

\section{FBm Properties}

Continuous $\mathrm{fBm}$ of $\mathrm{H}$ parameter in $] 0 ; 1\left[\right.$, denoted $\mathrm{B}_{\mathrm{H}}(\mathrm{t})$, is defined as an extension of Brownian motion $\mathrm{B}(\mathrm{t})[1]$ : 


$$
\mathrm{B}_{\mathrm{H}}(\mathrm{t})-\mathrm{B}_{\mathrm{H}}(0)=\frac{1}{\Gamma\left(\mathrm{H}+\frac{1}{2}\right)}\left\{\int_{-\infty}^{\mathrm{t}}(\mathrm{t}-\mathrm{s})^{\mathrm{H}-1 / 2} \mathrm{~dB}(\mathrm{~s})-\int_{-\infty}^{0}(-\mathrm{s})^{\mathrm{H}-1 / 2} \mathrm{~dB}(\mathrm{~s})\right\} .
$$

$\Gamma$ is the gamma function and when $\mathrm{H}=1 / 2, \mathrm{fBm}$ reduces to Brownian motion.

From now on, we will focus on properties of discrete processes denoted $\mathrm{B}_{\mathrm{H}}[\mathrm{i}]$ where $\mathrm{i}$ is a discrete time index. With a starting value $\mathrm{B}_{\mathrm{H}}[0]=0, \mathrm{fBm}$ is zero mean, Gaussian and second order non stationary as attested by its variance law deduced from (1):

$$
\operatorname{Var}\left(\mathrm{B}_{\mathrm{H}}[\mathrm{i}]\right)=\sigma^{2} \mathrm{i}^{2 \mathrm{H}} \text {. }
$$

Var is the variance operator, and $\sigma^{2}$ is the variance of $\mathrm{fBm}$ for the time index $\mathrm{i}=1$. From (2) the autocorrelation function of the process follows [1]:

$$
r_{B_{H}}[i, j]=E\left(B_{H}[i] B_{H}[j]\right)=\frac{\sigma^{2}}{2}\left(|i|^{2 H}+|j|^{2 H}-|i-j|^{2 H}\right) .
$$

$\mathrm{E}$ is the expectation operator. Using time-frequency tools, it was shown that the averaged power spectral density of $\mathrm{fBm}$ is proportional to $|\omega|^{-1-2 \mathrm{H}}$ [7]. When considering discrete signals, there always will be aliasing problems.

$\mathrm{fBm}$ has no derivative, and thus its increments for a time lag $\mathrm{m}$ are of interest. They are named fractional Gaussian noises (fGn), denoted $\mathrm{G}_{\mathrm{m}}$, and defined as:

$$
\mathrm{G}_{\mathrm{m}}[\mathrm{i}]=\mathrm{B}_{\mathrm{H}}[\mathrm{i}]-\mathrm{B}_{\mathrm{H}}[\mathrm{i}-\mathrm{m}] \text {. }
$$

They are zero mean, Gaussian and stationary processes since their autocorrelation can be written as:

$$
r_{G_{m}}[k]=E\left(G_{m}[i] G_{m}[i+k]\right)=\frac{\sigma^{2}}{2 m^{2 H}}\left(|k+m|^{2 H}-2|k|^{2 H}+|k-m|^{2 H}\right) .
$$

Without lost of generality, the case $m=1$ will be considered in the following. fGn for $\mathrm{m}=1$ will be noted $\mathrm{G} 1$ and its autocorrelation function derived from (5) becomes:

$$
\mathrm{r}_{\mathrm{Gl}}[\mathrm{k}]=\frac{\sigma^{2}}{2}\left(|\mathrm{k}+1|^{2 \mathrm{H}}-2|\mathrm{k}|^{2 \mathrm{H}}+|\mathrm{k}-1|^{2 \mathrm{H}}\right) \text {. }
$$

The following remarks regarding this equation will be useful in section 4 to explain some results.

For $\mathrm{H}=0.5$, increments are uncorrelated and $\mathrm{fGn}$ is the white Gausssian noise process. For $\mathrm{H}<0.5$, increments are negatively correlated. For $\mathrm{H}>0.5$, they are positively correlated and the process is said to have long term memory since $\mathrm{r}_{\mathrm{Gm}}[\mathrm{k}]$ decays hyperbolically with the lag $\mathrm{k}$. It should be noticed that for $\mathrm{H} \geq 1 / 2$ the function 
$\mathrm{r}_{\mathrm{G} 1}[\mathrm{k}]$ is always nonnegative. This sequence is also decreasing and convex, i. e. second differences are positive. Finally, for $\mathrm{H}<1 / 2$, one gets $\mathrm{r}_{\mathrm{G} 1}[\mathrm{k}]<0$ for any integer $\mathrm{k} \neq 0[8]$.

\section{ML H Parameter Estimation}

There exist a lot of estimators of the $\mathrm{H}$ parameter [2-3]. Among all of these, the ML is of interest because of its asymptotical efficiency [5]. A ML estimation of the $\mathrm{H}$ parameter is developed in [4] based on the fGn. Here, we propose to perform it directly on the $\mathrm{fBm}$ data. First, let us define $\mathbf{f B m}$, the $\mathrm{fBm}$ vector composed of $\mathrm{N}$ samples. Since all the samples of $\mathbf{f B m}$ are jointly Gaussian distributed, their likelihood function LF parameterised by $\mathrm{H}$ and $\sigma^{2}$ is:

$$
\mathrm{LF}\left(\mathbf{f B m} ; H, \sigma^{2}\right)=\frac{1}{(2 \Pi)^{N / 2}|\mathbf{R}|^{1 / 2}} \exp \left(-\frac{1}{2} \mathbf{f B m}^{T} \mathbf{R}^{-1} \mathbf{f B m}\right) \text {. }
$$

$\mathrm{N}$ is the size of $\mathbf{f B m}$ and $\mathbf{R}$ is its $\mathrm{N} \times \mathrm{N}$ covariance matrix where each element $[\mathbf{R}]_{i, j}$ depends on the autocorrelation function as $[\mathbf{R}]_{\mathrm{i}, \mathrm{j}}=\mathrm{r}_{\mathrm{BH}}[\mathrm{i}, \mathrm{j}]$ as defined in equation (3).

The maximum of the log-likelihood function (LLF, the logarithm of equation 7) is to be found where constant terms are neglected:

$$
\operatorname{LLF}\left(\mathbf{f B m} ; \mathrm{H}, \sigma^{2}\right)=-\operatorname{Ln}|\mathbf{R}|-\mathbf{f B m} \mathbf{m}^{T} \mathbf{R}^{-1} \mathbf{f B m} .
$$

$\mathbf{R}$ is first decomposed as $\sigma^{2} \mathbf{R}^{\prime}$ and the derivative of the LLF with respect to $\sigma^{2}$ is calculated. The value found by letting the derivative go to zero is inserted in the LLF and gives the final function to maximise with respect to $\mathrm{H}$. The $\mathrm{H}$ estimator noted $\hat{\mathrm{H}}$ is:

$$
\hat{\mathrm{H}}=\underset{0<H<1}{\operatorname{Max}}\left\{-\operatorname{Ln}\left|\mathbf{R}^{\prime}\right|-\mathrm{N} \operatorname{Ln}\left(\frac{\mathbf{f B m}^{T} \mathbf{R}^{-1} \mathbf{f B m}}{\mathrm{N}}\right)\right\} .
$$

As $\mathbf{f B m}[0]$ is zero by convention, the first row and column of $\mathbf{R}^{\prime}$ are all zeros and must not be considered, otherwise $\mathbf{R}^{\prime}$ is singular. The Gauss-Jordan elimination algorithm is used to compute the inverse and determinant of $\mathbf{R}$ '.

This ML based estimator is tested on synthetic signals. In 1D, there exist two methods theoretically exact to synthesis fractional Brownian motion. The first one is the method based on the Choleski decomposition of the covariance function [4]. It requires high computational resources due to its complexity of $O\left(N^{2}\right)$. The second one is the circulant embedding method (CEM) [9]. Since based on the fast Fourier transform (FFT) algorithm, its complexity is only $O(N \log N)$. CEM is used in our experimental tests. 100 signals of 100 samples each were synthesised for three typical $H$ values: $H=0.2, H=0.5$, and finally $H=0.8$. Mean $\hat{H}$ values are compared to the true 
$\mathrm{H}$ given during the synthesis of the reference signals. The standard deviations are also estimated. Results are shown in table 1.

Table 1. Mean \pm standard deviations (std) of the ML H estimators based on $\mathrm{fBm}$ in the first column. Computing are based on 100 synthetic signals of 100 samples each for $\mathrm{H}=0.2,0.5$ and 0.8. Second column shows results when a block of 100 unknown samples (indexed from 50 to 149 ) is added in the middle of each signal.

\begin{tabular}{ccc}
\hline True $\mathrm{H}$ & a) $\hat{\mathrm{H}}$ & $\begin{array}{c}\text { b) } \hat{\mathrm{H}} \\
\text { missing data } \\
\text { Mean } \pm \text { std }\end{array}$ \\
\hline 0.2 & $0.197 \pm 0.048$ & $0.205 \pm 0.048$ \\
0.5 & $0.496 \pm 0.060$ & $0.501 \pm 0.059$ \\
0.8 & $0.796 \pm 0.058$ & $0.797 \pm 0.058$ \\
\hline
\end{tabular}

The quality of this estimator can be studied. First, the bias of the estimates is low. A bilateral Student $t$ test with a level of significance of 0.01 shows that these estimates are unbiased. In identical conditions, the bias could be as high as 0.3 for some other analysis methods [2]. The standard deviations of the estimates are close to the square root of the Cramer-Rao lower bound which is equal to 0.046, 0.059 and 0.057 for respectively $\mathrm{H}=0.2,0.5$ and 0.8 for 100 samples [6]. An unilateral hypothesis test with a significance level of 0.01 shows that the variances of the estimates are equal to the respective Cramer-Rao lower bounds. These results show that in this case, the ML approach is efficient for data length as short as 100 samples.

This ML estimator can be easily used for non uniform sampling periods or when some samples are unknown. As an example, two blocks of 50 samples each are separated by 100 unknown samples for a $\mathrm{fBm}$ signal. The size of covariance matrix is $100 \times 100$. Each element is computed using (3) where $\mathrm{i}$ and $\mathrm{j}$ are the position indexes of the known samples. Table 1 (b) shows the results. It can be noticed that the bias is still low and that the variance is nearly unchanged. Thus, an efficient ML estimate of the $\mathrm{H}$ parameter can be achieved for particular signals. Such cases arise when studying incomplete time series or for irregularly sampled 1D data.

\section{$4 \quad$ ML Prediction and Interpolation}

Two direct extensions of the above method can be derived, namely ML prediction and interpolation processed on the $\mathrm{fBm}$ signal itself. The prediction problem has been theoretically treated in [10] while the interpolation has not been considered. Here a practical study on true fBm data is carried out for prediction as well as for interpolation.

There are now three parameters to estimate: $H, \sigma^{2}$ and the value of the data to be found. The problem can be split into two parts: a ML H estimation is first carried out on the signal, then the value of the prediction or interpolation is computed with known $\mathrm{H}$. 


\subsection{Prediction}

Let $\mathbf{f B m}[\mathrm{N}+\mathrm{x}]$ for $\mathrm{x}>0$ be a sample to predict given the $\mathrm{H}$ parameter and the first $\mathrm{N}$ samples of the vector. The theoretical correlation $r[i, N+x]$ between $\mathbf{f B m}[i]$ and $\mathbf{f B m}[\mathrm{N}+\mathrm{x}]$ with $1 \leq \mathrm{i} \leq \mathrm{N}$ is deduced from (3) with $\mathrm{j}$ being replaced by $\mathrm{N}+\mathrm{x}$. The covariance matrix $\mathbf{R}$ of $\mathbf{f B m}$ is now an $(\mathrm{N}+1) \times(\mathrm{N}+1)$ square matrix. It is identical to the one for the ML $\mathrm{H}$ estimation problem except that there are a row and a column added after respectively the last row and column to take into account the correlation between $\mathbf{f B m}[\mathrm{i}]$ and $\mathbf{f B m}[\mathrm{N}+\mathrm{x}]$. $\mathbf{R}$ can be decomposed as previously in $\sigma^{2} \mathbf{R}$. The final function is maximised with respect to $\mathbf{f B m}[\mathrm{N}+\mathrm{x}]$. This result can be seen as the mean prediction. The standard deviation easily follows based on the knowledge of the LLF.

We have tested this method on the same synthetic signals as previously described. Five samples of a typical realisation for $\mathrm{H}=0.2,0.5$ and 0.8 are represented as shown in figure 1 . Ten regularly spaced predictions are estimated after the last sample.

Remarks regarding equation (6) stated in section 2 are necessary to explain the results. For $\mathrm{H}=0.5$, the ML mean estimate is equal to the last value of the signal. Indeed, as its increments are uncorrelated, the probability of an increase is equal to the probability of a decrease. For $\mathrm{H}=0.8$, the ML estimate follows the trend of the past signal because increments are positively correlated. The shape looks similar to a polynomial prediction. For $\mathrm{H}=0.2$, the estimate goes in the opposite direction because increments are negatively correlated. The standard deviation of the estimates follows a power law due to the fact that increments are zero mean with standard deviation proportional to the lag at the power $\mathrm{H}$. This dependence on $\mathrm{H}$ is clearly seen in figure 1 .

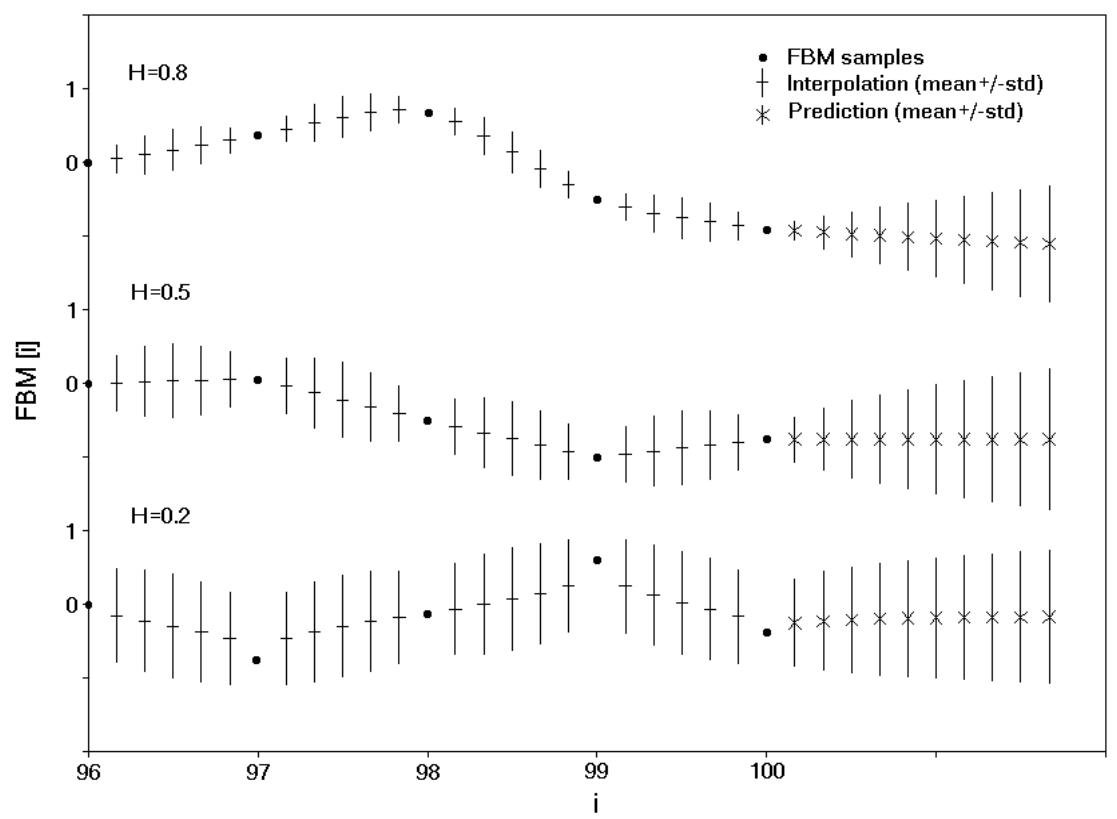

Fig. 1. Mean predictions and interpolations for a typical realisation of $\mathrm{fBm}$ for $\mathrm{H}=0.2,0.5$ and 0.8. The standard deviations of the estimates are also represented. 


\subsection{Interpolation}

Given the $\mathrm{H}$ parameter and $\mathrm{N}$ samples of the observation, the value to estimate is now $\mathbf{f B m}[\mathrm{k}+\mathrm{x}]$ for $1 \leq \mathrm{k}<\mathrm{N}$ and for $0<\mathrm{x}<1$. The correlation between $\mathbf{f B m}[\mathrm{i}]$ and $\mathbf{f B m}[\mathrm{k}+\mathrm{x}]$ is deduced from (3) with $\mathrm{j}$ being replaced by $\mathrm{k}+\mathrm{x}$. The covariance matrix $\mathbf{R}$ is a $(\mathrm{N}+1) \times(\mathrm{N}+1)$ square matrix with a row and a column added between respectively the $\mathrm{k}$ and $\mathrm{k}+1$ rows and columns. The same scheme as for the prediction process is applied. Figure 1 shows the results of five interpolations regularly spaced between each of the last five samples of the data.

For $\mathrm{H}=0.5$, the ML mean interpolation is a linear interpolation. This can be explained from the prediction results. Indeed, interpolation can be seen as a weighted combination of a forward prediction (knowing the k first samples) and of a backward prediction (knowing the samples from the $\mathrm{k}+1$ to the last one). Results for $\mathrm{H}=0.2$ and $\mathrm{H}=0.8$ can be identically explained. The standard deviations of the estimates are depending on $\mathrm{H}$ and on the distance from the nearest known sample.

\subsection{Discussion}

Prediction and interpolation for fractal signals can be applied to many real cases. One can mention financial domain to predict stock exchange or sub-pixel interpolation for fractal images. But, the above results enable a better understanding of conventional $\mathrm{fBm}$ synthesis techniques as the random midpoint displacement (MID) [11]. This iterative technique can be seen as a stochastic interpolation process. It consists in adding new points whose position along the horizontal axis is the middle of two adjacent points. The position on the vertical axis is given by a Gaussian random variable with mean equal to the average of the two adjacent points and with variance depending on $\mathrm{H}$. It is known that it fails to provide true $\mathrm{fBm}$ signals when $\mathrm{H} \neq 0.5$ [12]. Our results confirm this fact. For $\mathrm{H}=0.5$, the mean ML position (a linear interpolation) is identical to the one given during the MID synthesis (an average). But, for other $\mathrm{H}$ values, it is not true. A solution to improve the MID synthesis method keeping the same scheme would be as follows: replace the random variable of the MID generating process by a new one with mean value and variance given by the ML interpolation for $\mathrm{fBm}$ as described above.

\section{Conclusion}

In this communication, we have presented ML approaches performed on the $\mathrm{fBm}$ signal itself. On reference fractal signals, it has been shown that the ML method gave efficient results. It also allows to measure the $\mathrm{H}$ aparameter even when data are missing or when irregular sampling is present. Two direct extensions were derived concerning ML prediction and interpolation for $\mathrm{fBm}$ signals which could be of interest in many real cases. Results can be analysed taking into account the behaviour of the process for the various $\mathrm{H}$ values that were studied. They also explain 
approximations of fractal synthesis methods such as the midpoint displacement method.

Future work will concern the synthesis of true $2 \mathrm{D}$ fBm images buy using the new interpolation that is presented here. In addition the prediction of $1 \mathrm{D}$ signal as for stock exchange data will be a new and interesting application.

\section{References}

1. Mandelbrot, B.B., Van Ness, J.W.: Fractional Brownian Motion, Fractional Noises and Applications. SIAM 10(4), 422-438 (1968)

2. Gache, N., Flandrin, P., Garreau, D.: Fractal Dimension Estimators for Fractional Brownian Motion. In: Proceedings of the ICASSP, vol. 5, pp. 3557-3560 (1991)

3. Jennane, R., Harba, R., Jacquet, G.: Quality of Synthesis and Analysis Methods for Fractional Brownian Motion. In: Proceedings of the IEEE Workshop on Digital Signal Processing, pp. 307-310 (1996)

4. Lundahl, T., Ohley, W.J., Kay, S.M., Siffert, R.: Fractional Brownian Motion: A Maximum Likelihood Estimator and its Application to Image Texture. IEEE Transactions on Medical Imaging 5(3), 152-161 (1986)

5. Dahlhaus, R.: Efficient parameter estimation for self-similar processes. The Annals of Statistics 17, 1749-1766 (1989)

6. Hoeffer, S., Kumaresan, R., Pandit, M., Ohley, W.J.: Estimation of the Fractal Dimension of a Stochastic Fractal from Noise Corrupted Data. Archiv fuer Electronic und Übertragungstechnick 46(1), 13-21 (1992)

7. Flandrin, P.: On the Spectrum of Fractional Brownian Motions. IEEE Trans. on Info. Theory 35, 197-199 (1989)

8. Beran, J.: Statistics for Long-Memory Processes. Chapman \& Hall (1994)

9. Perrin, E., Harba, R., Jennane, R., Iribaren, I.: Fast and exact synthesis for 1D fractional Brownian motion and fractional Gaussian noises. IEEE Signal Processing Letters 9(11), 382-384 (2002)

10. Gripenberg, G., Norros, I.: On the prediction for fractional brownian motion. Journal of Applied Probabilities 33, 400-410 (1996)

11. Peitgen, H.O., Saupe, D. (eds.): The Science of Fractal Images. Springer, New York (1988)

12. Mandelbrot, B.B.: Comment on Computer Rendering of Fractal Stochastic Models. Communications of the ACM 25, 581-583 (1982) 